needed to explore the interactions between patient, carer and clinician perceptions of non-medical devices and, ultimately whether this influences the benefits for patient self-management of chronic breathlessness.

\section{IMPROVING THE IDENTIFICATION AND MANAGEMENT OF CONSTIPATION ON THE PALLIATIVE CARE UNIT, WANSBECK GENERAL HOSPITAL}

Elizabeth Marsh, Katie Frew, Emma McDougall. Palliative Care Unit, Wansbeck General Hospital

10.1136/spcare-2021-PCC.145

Background In 2020 the palliative care team at the inpatient palliative care unit at Wansbeck General Hospital identified the notable burden of constipation on patients, and the barrier to symptom improvement it caused. Our team designed a quality improvement project to identify and tackle this issue.

Aims Our team aimed to assess the documentation, identification and management of constipation on the unit. This was undertaken via ${ }^{1}$ instigating health care education, and (2) developing a patient information leaflet to empower patient's control of constipation.

Methods A 8 month QI project was undertaken on the unit, assessing the written and NerveCentre, $\phi$ documentation of bowels, prior to and following the instigation of staff education and patient leaflets, promoting patient lead documentation of their constipation. This project followed several PDSA cycles and identified an initial absence of patient perception of autonomy over constipation, as well as prescriber's limited knowledge of the regional guidance. The data from the measures gathered continuously using an electronic pro forma, with alternations following each cycle with further staff and patient education, and alternations to the language of the leaflets. A flow chart was generated following the measures indicating response to intervention.

Conclusion The prevalence of overall constipation halved from 46 to $22 \%$, with an increase on the NerveCentre documentation of $19 \%$. In those for whom constipation was appropriately identified $100 \%$ used the regional guidance to manage this. It was noted however that following the initial spike of identification of constipation this deteriorated after the change was instigated, demonstrating the need for on-going education to both patients and staff.

\section{WHAT ENABLES AND CONSTRAINS SHARED DECISION- MAKING ABOUT OPIATES FOR PEOPLE LIVING WITH CHRONIC BREATHLESSNESS? A SYSTEMATIC NARRATIVE REVIEW AND FRAMEWORK ANALYSIS OF PUBLISHED DATA USING A PROGRAM THEORY OF SHARED DECISION-MAKING}

Giovanna Impelliziere Licastro, Aliya Syahreni Prihartadi, Mark Pearson. Hull York Medical School, INSPIRE, Wolfson Palliative Care Research Centre, University of Hull

\subsection{6/spcare-2021-PCC.146}

Background Despite the evidence supporting the use of opioids to manage chronic breathlessness, these are rarely prescribed, indicating the need for enhanced SDM in this field. The Waldron 2020 SDM program theory explores the relationship between various factors in patients' and healthcare professionals' (HCPs) engagement in SDM. This systematic narrative literature review assesses enablers and constrainers of SDM in the prescription of opioids for chronic breathlessness and evaluates whether the Waldron'-SDM program theory can explain the operation of these factors.

Methods A literature search was conducted on Medline, Embase, PsychInfo, Cochrane Database of Systematic Reviews, CINAHL, Scopus, and Web of Science (1946 “June 2020). Studies were selected if they took place in Europe, Canada, or Oceania, and addressed an element present in decision making for the management of chronic breathlessness with opioids. Thematic analysis identified patient and HCP factors involved in the prescribing of opioids for chronic breathlessness. Their effect on SDM was assessed using Waldron's SDM programme theory.

Results Six themes were identified: concerns with side effects, knowledge of opioid use for breathlessness, acceptability, prior experiences, awareness of symptom severity, and system support. HCPs were reported to have more negative attitudes towards opioid use for breathlessness, particularly in the context of COPD, than patients. HCP's support by the MDT improved engagement in SDM. Open, clear communication addressing patients' questions and concerns, alongside HCP's revisiting of the discussion enabled SDM. A simple change in attitude from either the patient or HCP could be determining to increasing engagement with SDM.

Conclusions Research is scarce on SDM in opioid prescription for breathlessness. Limitations include the lack of quality appraisal of evidence. The Waldron SDM program theory appropriately supports SDM in this context. This review informs patient-centred care on management of chronic breathlessness with opioids through SDM, and highlights gaps in the literature.

\section{BALANCING PATIENT BENEFIT AND RISK OF RESISTANCE: AN AUDIT OF HOSPICE ANTIMICROBIAL PRESCRIBING}

Jasmine Cannon, Robert McConnell, Jolene Brown, Andrew Hughes. St Oswald's Hospice, Gosforth, Newcastle upon Tyne

\subsection{6/spcare-2021-PCC.147}

Background Antimicrobial stewardship aims to improve the safety and quality of patient care and reduce the risk of antimicrobial resistance. Symptoms of infection are often part of the natural deterioration towards end of life. Clinical evidence for the benefit of antimicrobials at the end-of-life is scarce and results inconclusive. Within our hospice, our aim of prescribing effectively whilst observing the principles of advance care planning led to an audit of practice as part of our antimicrobial stewardship.

Methods A retrospective audit was completed of all patients admitted over 3 months, where antimicrobials had been prescribed, considering; whether antimicrobials were prescribed in accordance with local trust guidelines; presence of indication, review and stop dates on each prescription and whether advance discussions were documented in relation to the use of antimicrobials.

Results Of 56 admissions identified, 23 (41\%) patients had 37 individual antimicrobials prescribed. The commonest sources of infection were chest (18), urinary tract (4), skin and soft tissue (4). 